\title{
Examining Relationships between Multiple Self-Reported Sleep Measures and Gait Domains in Cognitively Healthy Older Adults
}

\author{
John P.K. Bernstein ${ }^{a}$ Alyssa De Vito ${ }^{a}$ Daniel S. Weitzner ${ }^{a}$ Rebecca MacAulay ${ }^{b}$ \\ Matthew Calamiaa, c Robert Brouillette ${ }^{c}$ Heather Foil $^{c}$ Owen T. Carmichael ${ }^{d}$ \\ Jeffrey N. Keller ${ }^{\mathrm{C}}$ \\ a Department of Psychology, Louisiana State University, Baton Rouge, LA, USA; ${ }^{b}$ Department of Psychology, \\ University of Maine, Orono, ME, USA; ${ }^{C}$ Institute for Dementia Research and Prevention, Pennington Biomedical

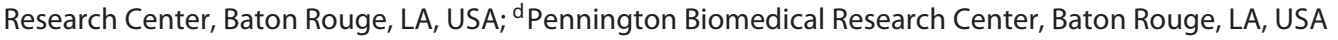

\author{
Keywords \\ Sleep · Gait · Older adults · Executive functioning
}

\begin{abstract}
Background: Gait-related changes in older adulthood may be related to changes in cognition (e.g., executive functioning), and recent work suggests that different self-reported measures of sleep may be tied to contrasting aspects of executive functioning. However, the relationship between these self-reported sleep measures and gait domains has not been explored. Such an investigation would be useful in helping to determine which older adults might exhibit changes in gait as well as experience other gait-associated changes (e.g., increased fall risk). Objective: To examine associations between different aspects of self-reported sleep and gait domains in a sample of cognitively healthy older adults. Method: A total of 423 older adults (mean age 69.9 years, range 50-92) completed self-report measures of sleep quality, daytime sleepiness, and sleep-related distress.
\end{abstract}

The participants also completed an objective, electronic measure of both single-task and dual-task gait (i.e., GAlTRite). Principal component analyses were used to elucidate the solution for each gait condition, and multiple linear regression was used to examine the contributions of sleep measures to variability in gait performance. Results: A 5-component solution of the single-task condition and a 4-component solution of the dual-task condition were identified. Multiple linear regressions revealed that a poorer sleep quality was associated with greater single-task and dual-task asymmetry. Greater daytime sleepiness was associated with increased dual-task gait variability and postural control. After controlling for the effects of other facets of sleep, sleep-related distress was not associated with any gait domain. Conclusions: Among cognitively healthy older adults, sleep quality and daytime sleepiness, but not sleeprelated distress, are associated with aspects of gait. Patients who report these symptoms should be assessed and monitored for possible changes in gait.

(c) 2019 S. Karger AG, Basel

\section{KARGER}

(c) 2019 S. Karger AG, Basel

E-Mail karger@karger.com

www.karger.com/ger
John P.K. Bernstein

Department of Psychology, Louisiana State University 217 Audubon Hall

Baton Rouge, LA 70803 (USA)

E-Mail jberns3@1su.edu 


\section{Introduction}

Changes to sleep architecture (e.g., sleep fragmentation and greater sleep onset latency) characterize normal aging and have been linked to a high prevalence of subjective sleep complaints among older adults [1]. Specifically, approximately $75 \%$ of older adults over the age of 65 report at least 1 sleep complaint [2]. These complaints become more common throughout the course of older adulthood as, in comparison to younger old adults (i.e., age 65-74 years), old-old adults (ages 75 and greater) are more likely to report symptoms of insomnia [3]. Subjective reports of differing aspects of poor sleep have been linked to poorer cognitive outcomes, with decrements observed in different aspects of neuropsychological functioning such as attention, memory, and executive functioning (EF) $[1,4]$ as well as the potential for an increased risk of cognitive decline [5]. In the oldest-old, age appears to exacerbate the effects of poor sleep on cognitive performance compared to other older adult age subgroups [6]. Poor sleep has also been linked to worse functional outcomes such as mental health diagnoses (e.g., anxiety and depression) [7], decreased physical health, and increased mortality in older adults [8].

Gait, or one's manner of walking (e.g., pace and rhythm), is central to older adults' mobility and serves as a strong predictor of fall risk [9]. Besides age-related somatic comorbidities such as osteoarthritis, polyneuropathy, and peripheral vascular diseases, other health conditions as well as psychological factors including cognition (e.g., attention and EF) and mood have been shown to be predictive of gait changes and associated negative outcomes [10]. More recently, sleep problems have been identified as another important factor that contributes to gait problems [11, 12]. Given these findings, researchers have suggested that several aspects of cognitive functioning necessary for gait control are the same ones that frequently deteriorate following alterations to sleep quality or quantity $[11,13]$. However, most studies have only examined relationships between sleep and gait speed rather than examining individual aspects of gait. Research in this area has shown that poor objectively measured sleep is associated with a slower gait speed $[10,14]$ as well as increased variability in stride length [10]. Findings from studies that examine the relationship between self-reported sleep and gait mirror work that utilized objective measurements of sleep, suggesting that poor sleep is associated with a slower gait speed, an abnormal cadence, and changes in stride variability $[12,15]$. Given that different aspects of self-reported sleep are differentially linked to important aspects of cognition that may underlie gait, such as attention and EF [4], further research into the relationship between different aspects of self-reported sleep and gait is warranted. Moreover, while objectively measured sleep and self-reported sleep are not consistently highly correlated, both have been linked to gait domains $[11,12,14,15]$.

Two types of gait assessments are typically performed in older adults, i.e., single-task (ST) gait assessments and dual-task (DT) gait assessments. ST assessments often involve walking only, whereas DT assessments involve walking while performing another task simultaneously such as spelling a word backwards [10]. Several studies have suggested that dual tasking requires greater attentional and $\mathrm{EF}$ resources than single tasks. Studies have found that this is especially true in older adults, given the fact that older adults rely more heavily on cognitive control to coordinate their balance and movement to compensate for sensory deficits that often decline in older age [16]. Importantly, DT situations are more common in daily life than ST situations and have been associated with a higher fall risk [10]. Therefore, older adults are at a higher risk for gait disturbance and falls during distracted conditions that are common in daily life, such as walking and talking to a friend, because their attention is divided. Further, recent evidence suggests a relationship between objective sleep quality and performance in DT but not ST assessment [10].

The current study aimed to expand the literature on the relationship between sleep and gait by examining relationships between different aspects of gait and 3 different measures of self-reported sleep in a large, cognitively healthy sample of older adults. Further, this study explores these different relationships in both ST and DT conditions. These findings may contribute to understanding of the sleep-gait relationship and improve the identification of older adults with an increased risk of gait disorders.

\section{Materials and Methods}

\section{Participants}

The participants of the current study were recruited from the Louisiana Aging Brain Study (LABrainS). The LABrainS is a longitudinal study of cognitive aging conducted by the Institute for Dementia Research and Prevention at the Pennington Biomedical Research Center. The participants of the LABrainS receive annual cognitive and mobility evaluations in order to assess longitudinal changes in cognition and mobility as well as assist in the improvement of clinical and research measures [17]. In total, over 40 of the 64 parishes that constitute Louisiana are represented in the LABrainS sample. In order to be included in LaBrainS, participants
Bernstein et al. 
must be older adults (i.e., aged 50 years or older) without cognitive impairment (i.e., Mini-Mental State Exam [MMSE] score of 25 or above). Participants must also not report clinically significant depression (i.e., score $>6$ on the 15 -item Geriatric Depression Scale [GDS]). LaBrainS participants who fail to meet these criteria at any study visit are excluded from further participation.

In order to be included in the present study, participants had to have complete sleep measures and have completed the GAITRite ST and DT conditions. Participants were also required to not have been diagnosed with a psychiatric or neurological disorder in the previous year in order to maintain a focus on healthy aging [18]. In this study, participants were excluded on the basis of a recent stroke $(n=3)$, transient ischemic attack $(n=3)$, traumatic brain injury $(n=8)$, a Parkinson's disease diagnosis $(n=1)$, or current alcohol or drug abuse $(n=3)$. Those who presently took medication for these disorders and other conditions that may affect cognitive performance were also excluded (i.e., anxiety, $n=24$; depression, $n=20$; muscle relaxant, $n=7$; central nervous system stimulant, $n=2$; Parkinson's disease, $n=1$; epilepsy/seizure, $n=7$; and pain from damaged nerves, $n=1)$. Participants who took prescribed medications for sleep disorders $(n=6)$ were excluded on the basis of an established relationship between sleep medication use and cognitive functioning (e.g., insomnia medication) [19]. Participants who did not complete a GaitRite assessment during the study year $(n=589)$ were also excluded. Participants may have been included in more than 1 exclusion group. The current study sample included many of the same participants whose data was analyzed in a prior paper on sleep and cognition [4].

Study visits were conducted by trained supervised research assistants, which included obtaining oral and written informed consent, completing cognitive and GaitRite assessments, and collecting health history information to ensure participants' continued eligibility for study inclusion.

\section{Measures}

Gait Assessment

The GAITRite system (CIR Systems, Inc., Sparta, NJ, USA) was used to objectively assess walking gait. The GAITRite is an electronic carpeted walkway (overall dimensions: $90 \mathrm{~cm} \times 700 \mathrm{~cm} \times$ $3.2 \mathrm{~mm}$ ) with pressure sensors that enables collection of data related to walking gait (e.g., step time and cadence). The GAITRite has been shown to be a valid means of assessing gait-related domains and it has been used in prior studies recruiting cognitively healthy older adults $[17,20]$.

Gait variables included in the analyses were selected based on prior work exploring particular aspects of gait in older adult populations as well as previous factor analyses of GAITRite outcome variables in these populations $[21,22]$. These included: step velocity, step length, step time variability, step swing time variability, step stance time variability, step time, swing time, step stance time, step time asymmetry, step swing time asymmetry, step stance asymmetry, step velocity variability, step length variability, and step length asymmetry. These data were collected over the course of a total of 4 trials, which included 2 ST trials followed by 2 DT trials. Participants were instructed to complete the trials "using their everyday walking pace." For each of the DT trials, consistent with previous research, participants were instructed to spell a 5-letter word backwards aloud read from a counterbalanced word list as they walked [23]. In order to avoid practice effects, participants were not assigned the same word on subsequent DT trials.

\section{Sleep Assessment}

This study included 3 widely used subjective sleep measures in order to examine the independent contributions of day- and nighttime sleep problems to aspects of gait. The Epworth Sleepiness Scale (ESS) was used to assess sleepiness experienced during the day and the likelihood of falling asleep in a variety of sedentary activities and settings over the previous 2 weeks [24]. The ESS includes 8 items rated on a 4-point Likert scale from 0 to 3 , with higher scores indicating greater sleepiness. Item scores are then summed, with total scores above 10 indicating significant sleepiness [25]. The Insomnia Severity Index (ISI) was used to examine the extent to which sleep-related problems have contributed to distress and impaired daily functioning over the previous 2 weeks [26]. The ISI includes 7 items rated on a 5 -point Likert scale from 0 to 4 , with higher scores indicating greater sleep-related distress and impairment. Item scores are then summed, with total scores between 0 and 7 indicating an absence of insomnia, scores of 8-14 indicating subthreshold insomnia, scores of 15-21 indicating moderate insomnia, and scores of 22-28 indicating severe insomnia [27]. The Pittsburgh Sleep Quality Index (PSQI) was used to assess sleep quantity, quality, and disturbances over the previous month. The PSQI is a 19-item measure with a mixture of open questions and 4-point Likert scale items scored from 0 to 3. Seven item scores are derived from the measure and then summed, with scores above 5 indicating a poor sleep quality [27]. The present study employed a modified version of the PSQI that did not include in analyses data regarding daytime sleepiness due to the study's goal of assessing sleep quality independently from daytime sleepiness [28]. Total scores, and not any subscale scores, were utilized as values.

\section{Depressive Symptom Assessment}

Depressive symptoms were assessed using the GDS, a fifteenitem measure of self-reported depressive symptoms in which larger scores indicate greater depression [29]. As noted previously, participants were excluded from LaBrainS if they obtained a score of 6 or higher on this measure, which is indicative of clinically significant depression [30]. As such, all participants included in analyses obtained scores of between 0 and 5 .

\section{Analyses}

SPSS version 25.0 was used for all statistical analyses. Disagreement persists in the literature regarding the number of components that comprise dual-task gait and which components of gait may be most important $[21,31,32]$. In order to reduce the number of gait variables included in analyses and create interpretable composites of gait, principal component analysis (PCA) was used as a statistical data reduction technique. Consistent with prior work exploring the principal components of GaitRite data in older adults and evidence that gait domains are generally not associated in older adults [21], a varimax rotation was chosen. A PCA was run for both a 4-component and 5-component solution in ST and DT conditions given previous work [21, 32]. Based on the results of the PCA, component scores were next saved and utilized as dependent variables in regressions.

Hierarchical multiple regressions were used to examine associations of sleep quality (i.e., modified PSQI), daytime sleepiness (i.e., ESS), and insomnia severity (i.e., ISI) with gait components. Given the goal of examining the contributions of day- and nighttime aspects of sleep to gait components, PSQI and ESS scores were entered in step 1 and ISI scores were entered in step 2 of regressions. This method allowed examination of the contributions of sleep-related distress and impairment to gait components after 
Table 1. PCA results for ST gait

\begin{tabular}{|c|c|c|c|c|c|}
\hline Step velocity & -0.48 & -0.13 & -0.02 & 0.84 & -0.02 \\
\hline Step length & 0.13 & -0.07 & -0.05 & 0.95 & 0.01 \\
\hline Step time variability & 0.12 & 0.75 & 0.14 & -0.04 & 0.01 \\
\hline Step swing time variability & 0.04 & 0.86 & -0.02 & -0.05 & 0.11 \\
\hline Step stance time variability & 0.09 & 0.85 & 0.00 & -0.08 & 0.06 \\
\hline Step stance time & 0.90 & 0.16 & 0.03 & -0.32 & 0.04 \\
\hline Step time asymmetry & 0.05 & 0.07 & 0.03 & -0.03 & 0.87 \\
\hline Step swing time asymmetry & -0.00 & 0.10 & -0.02 & -0.03 & 0.92 \\
\hline Step stance asymmetry & -0.06 & -0.06 & 0.01 & 0.00 & -0.92 \\
\hline Step velocity variability & -0.06 & 0.17 & 0.89 & 0.00 & -0.01 \\
\hline Step length variability & 0.05 & -0.02 & 0.90 & -0.09 & 0.02 \\
\hline
\end{tabular}

Values in bold indicates a significant loading.

sleep quality and daytime sleepiness have been accounted for [4]. Demographic variables (e.g., age, gender, and education) were also entered into step 1 regressions as these factors have been proven to be related to gait outcomes and physical activity more broadly among cognitively healthy older adults [33].

\section{Results}

\section{Sample Demographics}

Of the 1,029 individuals who completed LaBrainS visits during the study year, 423 met all of the inclusion and exclusion criteria and were included in the analyses. Among the participants $66.4 \%$ were female, $95.3 \%$ were white, and $1.4 \%$ were of Hispanic or Latino descent. The participants were an average of $69.9(\mathrm{SD}=7.6)$ years of age and had an average of $16.1(\mathrm{SD}=2.5)$ years of education. The participants had an average MMSE score of 29.5. In total, $18.0 \%$ of the participants endorsed significant sleepiness on the ESS, 7.8\% reported moderate or severe insomnia on the ISI, and $11.2 \%$ reported poor sleep quality on the PSQI.

\section{Primary Component Analysis}

The results of the ST PCA are shown in Table 1. By comparison to the 4-component solution, the 5-component solution yielded a component structure more similar to that identified in prior work examining ST GaitRite performance [21], and thus a 5-component solution was chosen to represent the ST measures. These components were named: (1) ST rhythm, (2) ST variability, (3) ST pace variability, (4) ST pace, and (5) ST asymmetry. ST rhythm refers to the overall gait cadence and timing. ST variability refers to stride-to-stride fluctuations in cadence and timing. ST pace variability refers to stride-to-stride fluctuations in pace. ST pace refers to the overall speed and length of steps. ST asymmetry refers to the degree of leftright limb coordination. Step length asymmetry did not load significantly onto any ST component. This 5-component solution accounted for $78.1 \%$ of the total variance.

The results of the dual-task PCA are shown in Table 2. By comparison to the 5-component solution, the 4-component solution yielded a component structure of the DT condition with fewer cross-loadings, thus offering a more distinctive representation of the DT measures. As such, the 4-component solution was chosen to represent the DT measures. These components were named:(1)DT rhythm/ variability, (2) DT pace variability/postural control, (3) DT pace/rhythm, and (4) DT asymmetry. DT rhythm/ variability refers both to overall and stride-to-stride fluctuations in cadence and timing. DT pace variability/postural control refers to stride-to-stride fluctuations in pace, as well as postural responses and sway. DT pace/rhythm refers to the overall speed and length of steps, as well as the overall gait cadence and timing. DT asymmetry refers to the degree of left-right limb coordination. This 4-component solution accounted for $78.5 \%$ of the total variance.

\section{Correlations among Sleep Measures}

Means, SD, medians and IQR information for demographic data, sleep measures, and gait variables is shown in Table 3. Daytime sleepiness and insomnia severity 
Table 2. PCA results for DT gait

\begin{tabular}{|c|c|c|c|c|}
\hline Aspect of gait & $\begin{array}{l}\text { Rhythm/ } \\
\text { variability }\end{array}$ & $\begin{array}{l}\text { Pace variability/ } \\
\text { postural control }\end{array}$ & Pace/rhythm & Asymmetry \\
\hline Step velocity & -0.27 & -0.06 & 0.95 & 0.02 \\
\hline Step length & 0.06 & 0.03 & 0.88 & 0.05 \\
\hline Step time variability & 0.77 & 0.19 & -0.22 & -0.00 \\
\hline Step swing time variability & 0.90 & 0.03 & 0.04 & -0.25 \\
\hline Swing time & 0.89 & 0.16 & -0.04 & -0.12 \\
\hline Step stance time & 0.29 & 0.21 & -0.69 & 0.14 \\
\hline Step time asymmetry & 0.36 & -0.14 & -0.02 & -0.68 \\
\hline Step swing time asymmetry & -0.22 & -0.04 & -0.10 & 0.91 \\
\hline Step stance asymmetry & 0.25 & -0.04 & -0.08 & -0.80 \\
\hline Step velocity variability & 0.04 & 0.88 & -0.09 & 0.05 \\
\hline
\end{tabular}

Values in bold indicate a significant loading.

Table 3. Descriptive statistics

\begin{tabular}{|c|c|c|c|c|}
\hline Variable & Mean \pm SD & Median & IQR & Patients, $n(\%)$ \\
\hline \multicolumn{5}{|l|}{ Sample demographics } \\
\hline Age, years & $69.90 \pm 7.62$ & 69.00 & $64.75-74.25$ & \\
\hline Female gender & & & & $281(66.4)$ \\
\hline White race & & & & $403(95.3)$ \\
\hline Non-Hispanic ethnicity & & & & $417(98.6)$ \\
\hline \multicolumn{5}{|l|}{ Sleep measures } \\
\hline Adjusted PSQI & $4.50 \pm 3.25$ & 4.00 & $2.00-6.50$ & \\
\hline ESS & $7.04 \pm 3.91$ & 7.00 & $4.00-10.00$ & \\
\hline ISI & $6.12 \pm 5.30$ & 5.00 & $2.00-10.00$ & \\
\hline \multicolumn{5}{|l|}{ Gait measures } \\
\hline Step stance time variability, ms & $6.47 \pm 5.32$ & 4.95 & $2.12-9.19$ & \\
\hline Step time, ms & $539.37 \pm 49.90$ & 535.25 & $503.50-569.00$ & \\
\hline Swing time, $\mathrm{ms}$ & $380.92 \pm 31.94$ & 376.00 & $359.00-400.00$ & \\
\hline Step stance time, $\mathrm{ms}$ & $697.22 \pm 75.30$ & 692.00 & $641.38-740.38$ & \\
\hline Step time asymmetry, ms & $10.04 \pm 0.27$ & 10.02 & $9.85-10.20$ & \\
\hline Step swing time asymmetry, ms & $1.01 \pm 0.03$ & 1.01 & $0.99-1.02$ & \\
\hline Step stance asymmetry, ms & $9.98 \pm 0.16$ & 9.97 & $9.88-10.08$ & \\
\hline Step velocity variability, $\mathrm{m} / \mathrm{s}$ & $0.03 \pm 0.02$ & 0.03 & $0.01-0.04$ & \\
\hline Step length variability, $\mathrm{m}$ & $0.02 \pm 0.01$ & 0.01 & $0.01-0.02$ & \\
\hline Step length asymmetry, $\mathrm{m}$ & $0.01 \pm 0.00$ & 0.01 & $0.01-0.01$ & \\
\hline
\end{tabular}


were only modestly correlated $(r=0.21, p<0.01)$. Daytime sleepiness and sleep quality were not significantly correlated $(r=0.08, p>0.05)$; however, as noted in Materials and Methods, the measure of sleep quality used did not include an item specifically related to daytime sleepiness. Sleep quality and insomnia severity were strongly correlated $(r=0.77, p<0.01)$.

\section{Associations between Sleep Measures and Gait Components}

Poorer sleep quality was associated with greater ST asymmetry $(F[8,493]=24.33, t[501]=-2.57, \beta=0.16$, $p<0.05)$. Poorer sleep quality was also associated with greater DT asymmetry $(F[8,493]=14.76, t[501]=-2.62$, $\beta=0.17, p<0.01)$. Greater daytime sleepiness was associated with increased DT pace variability/postural control $(F[8,493]=3.23, t[501]=2.53, \beta=0.12, p<0.05)$. No other associations of daytime sleepiness or sleep quality with ST or DT gait components were found. After accounting for the effects of daytime sleepiness and sleep quality, insomnia severity did not account for additional variance in any ST or DT gait components.

\section{Discussion}

Sleep complaints have been shown to be related to decreased cognitive functioning in older adulthood, with particularly strong effects observed in individuals aged 75 years and older $[1,4]$. A small number of studies have demonstrated associations between poor subjective sleep and aspects of gait including gait speed, abnormal cadence, and changes in stride variability $[12,15]$. However, associations with other aspects of gait (e.g., step time, step length, and step stance time) have not been previously explored. Previous studies have also been inconsistent in their use of ST or DT conditions when measuring changes in gait. Given the contributions of EF to particular gait domains and the effects of poor sleep on older adults' EF, an understanding of how particular facets of sleep predict individual gait domains is warranted. Such an exploration would help clinicians to better determine which older adults may be at risk for demonstrating particular impairments to gait (e.g., rhythm and asymmetry), which in turn may be useful in treatment planning. The goal of this study was to examine the relationship between different aspects of self-reported sleep problems and dimensions of ST and DT gait performance.

Before examining the relationship of different aspects of self-reported sleep to gait, we reduced the number of gait variables into composites using PCA. Prior PCA studies of gait have varied in terms of the population studied (e.g., adults aged 70 years and older or individuals who have undergone surgery for hip fracture) [31,32], the specific gait scores included in analyses, and the type of rotation used in the PCA $[21,32]$. These differences may contribute to discrepancies in the results that have been found across studies; however, in general, it should be noted that studies typically find separate components for pace, rhythm, asymmetry, variability, and postural control. In the current study, these components as well as an additional component of pace variability was found. In addition, this is the first study to the authors' knowledge to run PCA utilizing a DT condition. The components that were identified for the DT condition were rhythm/variability, pace variability/postural control, pace/rhythm, and asymmetry. Thus, when using the criterion of considering the distinctiveness of new components (i.e., minimizing cross-loadings), the PCA of the ST condition resulted in 5 components and the PCA of the DT condition resulted in 4 components. There is a need for more studies using PCA to identify relatively independent aspects of gait so that the rich data captured by the GaitRite system can be more fully utilized.

Gait asymmetry is characterized as differences in movement on one side of the body compared to the other, such as taking longer steps with one leg compared to the other [34]. The current study demonstrated that poor subjective sleep quality was associated with greater gait asymmetry in both the ST and DT conditions. Therefore, although previous research has found increased gait asymmetry in clinical populations such as those diagnosed with Parkinson's disease [9], the current study suggests that gait asymmetry can occur in cognitively healthy older adults who experience poor sleep quality. Further, research suggests that gait asymmetry contributes to fall risk through its involvement in the development of "freezing of gait" in patients with Parkinson's disease [35]. Taken together, poor subjective sleep quality may put older adults at an increased risk for falls due to changes in their symmetrical gait patterns.

Prior research suggests that older adults who report sleep problems are at increased risk of falling when completing a dual-task gait measure [10]. However, findings of the current study suggest that even under ST conditions older adults with poor sleep quality may demonstrate gait asymmetry, which has been suggested as an important factor for predicting future falls [36]. Therefore, in cases where motor difficulties result in a referral for physical therapy, addressing issues related to sleep 
quality in conjunction can also be critical in helping to prevent falls in older adults.

In the current study, daytime sleepiness was associated with increased pace variability/postural control in the DT condition. Similarly, previous studies utilizing a DT condition have found that sleep deprivation negatively impacts postural control [37]. Further, a study of younger adults utilizing a dynamic test paradigm demonstrated that a lack of visual input negatively impacts postural balance in individuals with objectively measured chronic sleep problems [38]. Therefore, in conditions that require increased attentional capacity, problems with sleep are negatively associated with postural control.

The current study found that, after accounting for the effects of daytime sleepiness and sleep quality, insomnia severity did not account for any additional variance in any gait components in either ST or DT conditions. This is a surprising finding as previous work [4] has demonstrated that self-reported insomnia severity is tied to aspects of EF independently from daytime sleepiness. Furthermore, other work has noted that, in comparison to ST conditions, DT conditions are heavily reliant on intact EF [13].

Subtle changes in gait have been identified in individuals at risk for neurodegenerative disorders, such as Lewy body disease, prior to the emergence of significant motor or cognitive deficits [12]. Given that alterations in sleep quality and quantity may impact aspects of gait in old age, the assessment of sleep in this population may prove useful in predicting likelihood of functional decline. Furthermore, interventions that target sleep may have added health benefits in the forms of improved gait, a decreased fall risk, and a lower risk of developing cognitive impairment. Future work may elucidate these effects via longitudinal investigations examining the relationship between changes in aspects of sleep and facets of gait over the course of older adulthood.

\section{Limitations}

A limitation of the current study is the use of self-reported sleep rather than utilizing objective measures of sleep. Due to discrepancies between subjective and objective measures of sleep in older adults [39], future studies should utilize both types of measure to assess sleep characteristics that can impact changes in gait. A second limitation of the current study is the homogeneity of the sample. Many of the participants in the study were well-educated, financially secure, Caucasian women. Therefore, the findings of the current study may not be generalizable to individuals outside of this demographic range. The contributions of medication use and medical comorbidities rele- vant for sleep quality, while potentially relevant to the relationship between sleep and gait [40], were outside the scope of the present study and should be explored in future work. Finally, although some evidence suggests that gait deteriorates as a function of declines in cognitive functioning, others have noted that this relationship may be bidirectional [41]. Thus, changes in sleep quality in older adulthood may lead to alterations in gait due to sleep's effect on cognitive performance, but the effects of sleep may also impact cognition via its effect on physical functioning.

\section{Acknowledgement}

The authors thank the LaBrainS participants who made this work possible.

\section{Statement of Ethics}

All of the participants gave written informed consent. The study protocol was approved by the Pennington Biomedical Research Center Institutional Review Board.

\section{Disclosure Statement}

The authors have no conflict of interests to declare.

\section{Funding Sources}

This work was supported by National Institutes of Health grant R21AG044258 for "Walking and Cognitive Remediation Effects on Cerebrovascular Function and Gait" to Jeffrey Keller.

\section{Author Contributions}

All of the authors contributed substantially to this work as per guidelines in the ICMJE criteria for authorship.

References

1 Scullin MK, Bliwise DL. Sleep, cognition, and normal aging: integrating a half century of multidisciplinary research. Perspect Psychol Sci. 2015 Jan;10(1):97-137.

2 Fung CH, Vitiello MV, Alessi CA, Kuchel GA; AGS/NIA Sleep Conference Planning Committee and Faculty. Report and research agen$\mathrm{da}$ of the American Geriatrics Society and $\mathrm{Na}$ tional Institute on aging bedside-to-bench conference on sleep, circadian rhythms, and aging: new avenues for improving brain health, physical health, and functioning. J Am Geriatr Soc. 2016 Dec;64(12):e238-47. 
3 McCrae CS, Rowe MA, Tierney CG, Dautovich ND, Definis AL, McNamara JP. Sleep complaints, subjective and objective sleep patterns, health, psychological adjustment, and daytime functioning in communitydwelling older adults. J Gerontol B Psychol Sci Soc Sci. 2005 Jul;60(4):182-9.

4 Bernstein JP, Calamia M, Keller JN. Multiple self-reported sleep measures are differentially associated with cognitive performance in community-dwelling nondemented elderly. Neuropsychology. 2018 Feb;32(2):220-9.

5 Leng Y, McEvoy CT, Allen IE, Yaffe K. Association of sleep-disordered breathing with cognitive function and risk of cognitive impairment: a systematic review and meta-analysis. JAMA Neurol. 2017 Oct;74(10):1237-45.

6 Blackwell T, Yaffe K, Ancoli-Israel S, Schneider JL, Cauley JA, Hillier TA, et al.; Study of Osteoporotic Fractures Group. Poor sleep is associated with impaired cognitive function in older women: the study of osteoporotic fractures. J Gerontol A Biol Sci Med Sci. 2006 Apr;61(4):405-10.

7 Alvaro PK, Roberts RM, Harris JK. A systematic review assessing bidirectionality between sleep disturbances, anxiety, and depression. Sleep (Basel). 2013 Jul;36(7):1059-68.

8 Gallicchio L, Kalesan B. Sleep duration and mortality: a systematic review and meta-analysis. J Sleep Res. 2009 Jun; 18(2):148-58.

9 Yogev G, Plotnik M, Peretz C, Giladi N, Hausdorff JM. Gait asymmetry in patients with Parkinson's disease and elderly fallers: when does the bilateral coordination of gait require attention? Exp Brain Res. 2007 Mar;177(3): $336-46$.

10 Agmon M, Shochat T, Kizony R. Sleep quality is associated with walking under dual-task, but not single-task performance. Gait Posture. 2016 Sep;49:127-31.

11 Allali G, Perrig S, Cleusix M, Herrmann FR, Adler D, Gex G, et al. Gait abnormalities in obstructive sleep apnea and impact of continuous positive airway pressure. Respir Physiol Neurobiol. 2014 Sep;201:31-3.

12 McDade EM, Boot BP, Christianson TJ, Pankratz VS, Boeve BF, Ferman TJ, et al. Subtle gait changes in patients with REM sleep behavior disorder. Mov Disord. 2013 Nov; 28(13):1847-53.

13 Yogev-Seligmann G, Hausdorff JM, Giladi N The role of executive function and attention in gait. Mov Disord. 2008 Feb;23(3):329-42.

14 Goldman SE, Stone KL, Ancoli-Israel S, Blackwell T, Ewing SK, Boudreau R, et al. Poor sleep is associated with poorer physical performance and greater functional limitations in older women. Sleep. 2007 Oct;30(10): 1317-24.

15 Nakakubo S, Doi T, Shimada H, Ono R, Makizako H, Tsutsumimoto K, et al. The Association Between Excessive Daytime Sleepiness and Gait Parameters in CommunityDwelling Older Adults: Cross-Sectional Find- ings From the Obu Study of Health Promotion for the Elderly. J Aging Health. 2018 Feb; 30(2):213-28.

16 Hausdorff JM, Schweiger A, Herman T, Yogev-Seligmann G, Giladi N. Dual-task decrements in gait: contributing factors among healthy older adults. J Gerontol A Biol Sci Med Sci. 2008 Dec;63(12):1335-43.

17 MacAulay RK, Brouillette RM, Foil HC, Bruce-Keller AJ, Keller JN. A longitudinal study on dual-tasking effects on gait: cognitive change predicts gait variance in the elderly. PLoS One. 2014 Jun;9(6):e99436.

18 Moretti L, Cristofori I, Weaver SM, Chau A, Portelli JN, Grafman J. Cognitive decline in older adults with a history of traumatic brain injury. Lancet Neurol. 2012 Dec;11(12):110312.

19 Kleykamp BA, Griffiths RR, McCann UD, Smith MT, Mintzer MZ. Acute effects of zolpidem extended-release on cognitive performance and sleep in healthy males after repeated nightly use. Exp Clin Psychopharmacol. $2012 \mathrm{Feb} ; 20(1): 28-39$.

20 Bilney B, Morris M, Webster K. Concurrent related validity of the GAITRite walkway system for quantification of the spatial and temporal parameters of gait. Gait Posture. 2003 Feb;17(1):68-74.

21 Lord S, Galna B, Verghese J, Coleman S, Burn $\mathrm{D}$, Rochester L. Independent domains of gait in older adults and associated motor and nonmotor attributes: validation of a factor analysis approach. J Gerontol A Biol Sci Med Sci. 2013 Jul;68(7):820-7.

22 Brach IS, Perera S, Studenski S, Katz M, Hall $\mathrm{C}$, Verghese J. Meaningful change in measures of gait variability in older adults. Gait Posture. 2010 Feb;31(2):175-9.

23 Hollman JH, Kovash FM, Kubik JJ, Linbo RA. Age-related differences in spatiotemporal markers of gait stability during dual task walking. Gait Posture. 2007 Jun;26(1):113-9.

24 Johns MW. A new method for measuring daytime sleepiness: the Epworth sleepiness scale. Sleep. 1991 Dec;14(6):540-5.

25 Buysse DJ, Hall ML, Strollo PJ, Kamarck TW, Owens J, Lee L, et al. Relationships between the Pittsburgh Sleep Quality Index (PSQI), Epworth Sleepiness Scale (ESS), and clinical/polysomnographic measures in a community sample. J Clin Sleep Med. 2008 Dec;4(6):563-71.

26 Bastien $\mathrm{CH}$, Vallières A, Morin CM. Validation of the Insomnia Severity Index as an outcome measure for insomnia research. Sleep Med. 2001 Jul;2(4):297-307.

27 Morin CM, Belleville G, Bélanger L, Ivers H. The Insomnia Severity Index: psychometric indicators to detect insomnia cases and evaluate treatment response. Sleep (Basel). 2011 May;34(5):601-8.

28 Buysse DJ, Reynolds CF 3rd, Monk TH, Hoch CC, Yeager AL, Kupfer DJ. Quantification of subjective sleep quality in healthy elderly men and women using the Pittsburgh Sleep Qual- ity Index (PSQI). Sleep. 1991 Aug;14(4):3318.

29 Yesavage JA, Brink TL, Rose TL, Lum O, Huang V, Adey M, et al. Development and validation of a geriatric depression screening scale: a preliminary report. J Psychiatr Res. 1982-1983;17(1):37-49.

30 Sheikh JI, Yesavage JA. Geriatric Depression Scale (GDS): recent evidence and development of a shorter version. Clin Gerontol. 1986;5:165-73

31 Hollman JH, McDade EM, Petersen RC. Normative spatiotemporal gait parameters in older adults. Gait Posture. 2011 May;34(1):1118.

32 Thingstad P, Egerton T, Ihlen EF, Taraldsen K, Moe-Nilssen R, Helbostad JL. Identification of gait domains and key gait variables following hip fracture. BMC Geriatr. 2015 Nov; 15(1): 150 .

33 van Hooren SA, Valentijn AM, Bosma $H$, Ponds RW, van Boxtel MP, Jolles J. Cognitive functioning in healthy older adults aged 6481: a cohort study into the effects of age, sex, and education. Neuropsychol Dev Cogn B Aging Neuropsychol Cogn. 2007 Jan;14(1): 40-54.

34 Hodt-Billington C, Helbostad JL, Moe-Nilssen R. Should trunk movement or footfall parameters quantify gait asymmetry in chronic stroke patients? Gait Posture. 2008 May; 27(4):552-8.

35 Frazzitta G, Pezzoli G, Bertotti G, Maestri R. Asymmetry and freezing of gait in parkinsonian patients. J Neurol. 2013 Jan;260(1):71-6.

36 Hill K, Schwarz J, Flicker L, Carroll S. Falls among healthy, community-dwelling, older women: a prospective study of frequency, circumstances, consequences and prediction accuracy. Aust N Z J Public Health. 1999 Feb; 23(1):41-8.

37 Patel M, Gomez S, Berg S, Almbladh P, Lindblad J, Petersen H, et al. Effects of 24-h and 36-h sleep deprivation on human postural control and adaptation. Exp Brain Res. 2008 Feb;185(2):165-73.

38 Furtado F, Gonçalves BD, Abranches IL, Abrantes AF, Forner-Cordero A. Chronic low quality sleep impairs postural control in healthy adults. PLoS One. 2016 Oct;11(10): e0163310.

39 Van Den Berg JF, Van Rooij FJ, Vos H, Tulen $\mathrm{JH}$, Hofman A, Miedema HM, et al. Disagreement between subjective and actigraphic measures of sleep duration in a populationbased study of elderly persons. J Sleep Res. 2008 Sep;17(3):295-302.

40 A Tahrani A. Obstructive sleep apnoea and vascular disease in patients with type 2 diabetes. Eur Endocrinol. 2015 Aug;11(2):81-9.

41 Clouston SA, Brewster P, Kuh D, Richards M, Cooper R, Hardy R, et al. The dynamic relationship between physical function and cognition in longitudinal aging cohorts. Epidemiol Rev. 2013;35(1):33-50. 